\title{
Optimal Sliding Mode Control for Cutting Tasks of Quick-Return Mechanisms
}

\begin{abstract}
A solution of the constant cutting velocity problem of quick-return mechanisms is the main concern of this paper. An optimal sliding mode control in the task space is used to achieve uniform and accurate cuts throughout the workpiece. The switching hyperplane is designed to minimize the position error of the slider-dynamics in an infinite horizon.

A Jacobian compensator is used to exploit the mechanical advantage and ensure controllability. The velocity profile is constructed in terms of the mechanism and workpiece geometric properties. Stability of the closed-loop dynamics is verified with the Lyapunov stability theory. Experiments are carried out in a quick-return mechanism prototype to validate the proposal.

Keywords: Constant cutting velocity, Quick-return mechanism, Optimal sliding mode control, Slider dynamics, Jacobian compensator
\end{abstract}

\section{Introduction}

Many manufacturing applications using shapers, motorized saws[1], bombs [2] and so on; require a constant cutting velocity, $\dot{x}=v$, to ensure quality of the workpiece and reduce dimensionality errors $[3,4]$. The velocity $v \in \mathbb{R}$ denotes a desired constant cutting velocity. It has been shown in $[5,6]$ that the heat transfer and temperature of both the workpiece and the cutting tool are reduced and remain approximately constant when a constant cutting velocity is used. In consequence, uniform cuts and reduction of dimensional errors on the machined surface $[7,8]$ are obtained.

The main devices for cutting applications use some configuration of the 
quick-return mechanism $[9,10]$ such as Whitworth mechanism $[11,12,13]$, crank-shaper, power-driven saw [14], among others. These mechanisms provide to the reciprocating tool $^{1}$ (in this paper is denoted as the slider) with a slow cutting stroke and a quick return[15]. The cutting stroke presents high mechanical advantage [16], meanwhile the return stroke does not execute any task. The cutting stroke is where any cutting task is developed, hence is evident that it must be controlled to achieve uniform cuts.

The reciprocating $[1,2]$ and mechanism synthesis [17] methods are commonly used together to achieve an approximate constant cutting velocity, $\dot{x} \approx v$, at the cutting tool while using a fixed angular velocity, $\dot{q}=\omega(\omega \in \mathbb{R})$, at the mechanism-crank and a fixed mechanism configuration. However, uniform cuts throughout the workpiece cannot be ensured with this approach because it fails to consider the geometric dimensions of the mechanism and workpiece.

In view of the above, task-space controllers $[18,19,20]$ should be a good solution since the cutting task is developed in the task space. However, these controllers are avoided because they lose controllability at singularity points $[20,21]$, that is, the Jacobian loses rank. Furthermore, the contact between the slider and the workpiece generates an external force [22] which must be taken into account in the controller design $[23,24]$ by means of the virtual work principle.

\section{Related work}

Figure 1 illustrates the slider's phase diagram of any quick-return mechanism using the reciprocating method. A fixed angular velocity is applied at the mechanism crank such that the slider performs an approximate constant linear velocity. Any joint space controller can be used to control the mechanism dynamics [25]. When the slider velocity is not constant, non-uniform cuts are

\footnotetext{
${ }^{1}$ The reciprocating tool or mechanism end-effector is the slider which is located at the output link.
} 
obtained which affects the surface roughness of the workpiece and hence is not desirable for industrial applications [26, 27].

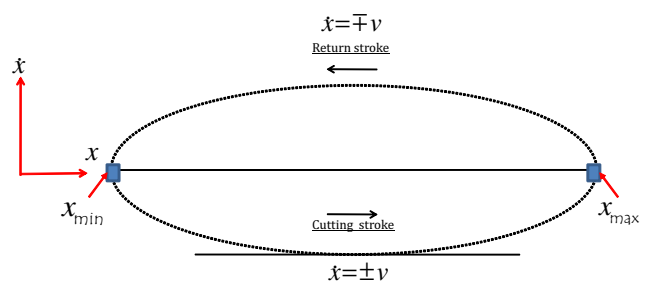

Figure 1: Slider's phase diagram using the reciprocating method

The main advantage of joint space controllers is that the controllability problem caused by the two well identified singularities of the quick-return mechanism is avoided [28]. The singularity points, where the transition between the cutting and return strokes is performed, are located at the beginning $x_{\min }=f\left(q_{\min }\right)$ and at the end $x_{\max }=f\left(q_{\max }\right)$ of the slider stroke. Here $f$ is the forward kinematics of the mechanism (see Appendix B) and $q_{\min }$ and $q_{\max }$ denote the inverse kinematics solution [29].

However, Figure 1 shows that the geometric dimensions of the slider stroke and workpiece are not considered. Moreover, the slider velocity is approximately constant only in a small position interval (see Figure 1). This issue can be solved by changing the input-crank velocities in a kinematic optimization problem $[2,11]$. Nevertheless, the singularities, the mechanical advantage, and the workpiece-mechanism interaction are not considered by this method [16] since it uses a joint space controller to control both strokes.

In summary, the main issues of previous related work are: (1) the reciprocating method achieves an approximate constant cutting velocity in a small time interval, (2) the mechanism synthesis method gives as output a fixed mechanism geometric configuration with low versatility, (3) joint space controllers do not consider geometric dimensions to develop the cutting task, (4) task-space controllers do not exploit the mechanical advantage in the singularity points, (5) both the cutting and return strokes are controlled. 


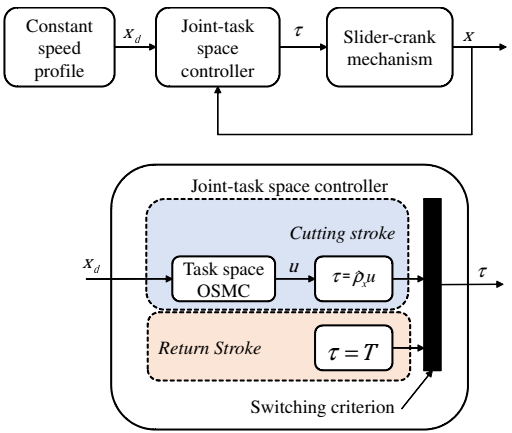

Figure 2: Proposed approach block scheme.

In this work, a solution of the constant cutting velocity issue of quick-return mechanism is proposed. Figure 2 shows the proposed approach block scheme. A second-order task space optimal sliding mode controller (OSMC) with a Jacobian compensator is used to control the cutting stroke. A fixed torque $\tau=T, T \in \mathbb{R}$ is used to control in open-loop the return stroke. A switching criterion is proposed to commute the OSMC into the fixed torque value and vice versa. The comparison and main contributions of this work are given in Table 1.

\section{Switching criterion design}

The slider's phase diagram of Figure 3 is proposed to enable the use of task-space controllers for cutting tasks applications of quick-return mechanisms without the controllability issue at the singularity points.

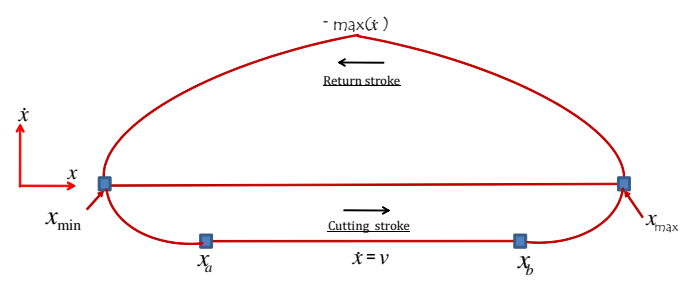

Figure 3: Slider's phase diagram of the proposed approach

The above Figure shows two new points $x_{a}$ and $x_{b}$. These points determine 
Table 1: Comparison of main contributions of the proposed approach for cutting applications

\begin{tabular}{|c|c|c|c|}
\hline Method and Controller & Motivation & Advantages & Disadvantages \\
\hline $\begin{array}{l}\text { Reciprocating and mechanism syn- } \\
\text { thesis methods (Joint space control) }\end{array}$ & $\begin{array}{l}\text { Achieve an approximate constant } \\
\text { cutting velocity using a fixed angu- } \\
\text { lar velocity at the mechanism crank. }\end{array}$ & $\begin{array}{l}\text { The mechanical advantage is ex- } \\
\text { ploited. The singularity points are } \\
\text { avoided. }\end{array}$ & $\begin{array}{l}\text { Both the cutting and slider stroke } \\
\text { are controlled. The velocity is ap- } \\
\text { proximately constant in a small po- } \\
\text { sition interval. The mechanism has } \\
\text { a fixed configuration. It does not } \\
\text { consider the length and type of ma- } \\
\text { terial of the workpiece. }\end{array}$ \\
\hline Task space control & $\begin{array}{l}\text { Achieve a constant cutting velocity } \\
\text { by controlling the slider directly in } \\
\text { the task space. }\end{array}$ & $\begin{array}{l}\text { The mechanism has a free configura- } \\
\text { tion. A constant cutting velocity is } \\
\text { achieved. The cutting velocity pro- } \\
\text { file is designed in accordance to the } \\
\text { geometric dimensions of the work- } \\
\text { piece and the slider stroke. }\end{array}$ & $\begin{array}{l}\text { The controller loses controllability } \\
\text { in the singularity points. The me- } \\
\text { chanical advantage is not exploited. }\end{array}$ \\
\hline $\begin{array}{l}\text { The proposed approach (Joint and } \\
\text { task space controller) }\end{array}$ & $\begin{array}{l}\text { Achieve a constant cutting velocity } \\
\text { by controlling the slider directly in } \\
\text { the task space and guarantee com- } \\
\text { plete turn of the mechanism crank. }\end{array}$ & $\begin{array}{l}\text { A constant cutting velocity is } \\
\text { achieved. The mechanism has a free } \\
\text { configuration. The mechanical ad- } \\
\text { vantage is exploited. The singular- } \\
\text { ity points are avoided. The cutting } \\
\text { velocity profile is designed in accor- } \\
\text { dance to the dimension of the work- } \\
\text { piece, type of material and cutting } \\
\text { velocity. }\end{array}$ & $\begin{array}{l}\text { It requires two controllers. Unde- } \\
\text { sired jerks in switching controllers. } \\
\text { The Jacobian compensator must be } \\
\text { designed off-line. }\end{array}$ \\
\hline
\end{tabular}

the length of the workpiece $L_{d}$, that is, $L_{d}=x_{b}-x_{a}$. The complete slider stroke $L_{d}$ is given by $L_{c}=x_{\max }-x_{\min }$. The switching criterion is applied at the singularity points and is defined as

$$
x_{\min }<x<x_{\max }
$$

The switching criterion (1) states that: if the slider position $x$ satisfies (1), then the task-space controller is applied; otherwise, the open-loop torque is applied, $\tau=T$, to return the mechanism with high velocity amplitude to the point $x_{\min }$.

The main issue of (1) is to recognize in which stroke is located the mechanism. A good solution is to use the sign of the slider velocity $z$ (this sign is obtained off-line) at any stroke and compare it with the sign of the current velocity such that the if-else condition of Figure 4 is satisfied.

\section{Constant Velocity Profile}

The proposed velocity profile considers the workpiece and slider stroke lengths, and the desired cutting velocity $v$. A trapezoidal trajectory with parabolic 


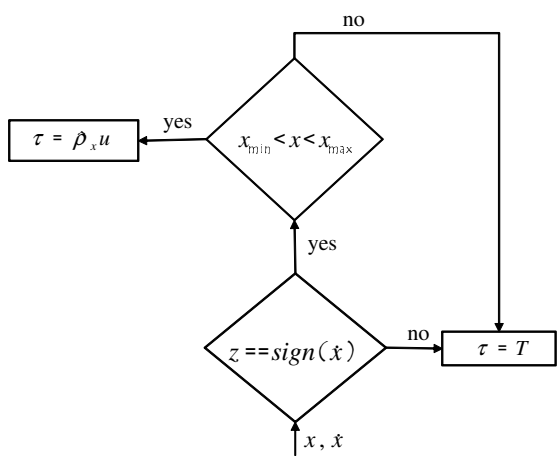

Figure 4: Switching criterion scheme.

blends is used as desired trajectory.

Let $t_{a}=t_{d}$ defines the times of the parabolic blends from $x_{\min }$ to $x_{a}$ and $x_{b}$ to $x_{\max }$ respectively, and $t_{c}$ denotes the cutting time from $x_{a}$ to $x_{b}$. Algorithm 1 exhibits a simple way to compute the points of the parabolic trajectory.

\section{Controller Design}

An OSMC is used to ensure velocity tracking and robustness against disturbances.

\subsection{Slider Linear Dynamics}

The extended dynamic model [30] of a 1-DOF quick-return mechanism is

$$
\boldsymbol{M}^{\prime}\left(\boldsymbol{q}^{\prime}\right) \ddot{\boldsymbol{q}}^{\prime}+\boldsymbol{C}^{\prime}\left(\boldsymbol{q}^{\prime}, \dot{\boldsymbol{q}}^{\prime}\right) \dot{\boldsymbol{q}}^{\prime}+\boldsymbol{G}^{\prime}\left(\boldsymbol{q}^{\prime}\right)=\boldsymbol{\rho}^{-\top}\left(\boldsymbol{q}^{\prime}\right)\left(\tau-\rho_{x}\left(\boldsymbol{q}^{\prime}\right) F_{x}\right)
$$

where $\boldsymbol{q}^{\prime}=[q, \boldsymbol{s}]^{\top} \in \mathbb{R}^{n^{\prime}}$ are the extended coordinates whose components are the generalized coordinate $q$ and all the $n$ secondary variables $s \in \mathbb{R}^{n}$, so $n^{\prime}=$ $n+1$. $\boldsymbol{M}^{\prime}\left(\boldsymbol{q}^{\prime}\right) \in \mathbb{R}^{n^{\prime} \times n^{\prime}}$ denotes a symmetric positive definite inertia matrix, $\boldsymbol{C}^{\prime}\left(\boldsymbol{q}^{\prime}, \dot{\boldsymbol{q}}^{\prime}\right) \in \mathbb{R}^{n^{\prime} \times n^{\prime}}$ stands for the centrifugal and Coriolis matrix, $\boldsymbol{G}^{\prime}\left(\boldsymbol{q}^{\prime}\right) \in \mathbb{R}^{n^{\prime}}$ is the gravity vector, $\boldsymbol{\rho}\left(\boldsymbol{q}^{\prime}\right) \in \mathbb{R}^{n^{\prime}}$ is the Jacobian vector in terms of $\boldsymbol{q}^{\prime}, \rho_{x}\left(\boldsymbol{q}^{\prime}\right)$ is the Jacobian component that gives the mapping between the joint velocity $\dot{q}$ and the slider velocity $\dot{x}, F_{x} \in \mathbb{R}$ is the slider contact force and $\tau \in \mathbb{R}$ is the control input. 


\section{Algorithm 1 Velocity profile parameters design \\ Require: Lengths $L_{d}, L_{c}$, constant velocity $v$.}

1: Obtain the joint positions $q_{\max }$ and $q_{\min }$ where the Jacobian loses rank, that is, $\rho_{x}(q)=0$.

2: Obtain $x_{\min }$ and $x_{\max }$ with $q_{\min }$ and $q_{\max }$, respectively; using the forward kinematics.

3: Calculate the trajectory times with

$$
\begin{aligned}
t_{c} & =\frac{L_{d}}{|v|} \\
t_{a} & =t_{d}=\frac{L_{c}}{|v|}-t_{c}
\end{aligned}
$$

4: The points $x_{a}$ and $x_{b}$ are obtained with

$$
\begin{aligned}
& x_{a}=x_{\min }+\frac{v}{2 t_{a}} t_{a}^{2} \\
& x_{b}=x_{\min }+\frac{v}{2 t_{a}} t_{a}^{2}+v t_{c}
\end{aligned}
$$

5: return $x_{a}, x_{b}, x_{\max }, x_{\min }, t_{a}, t_{d}$.

The slider dynamics of any quick-return mechanism can be expressed as a perturbed double integrator system [9] as

$$
m \ddot{x}+G=\left(\sum_{i=1}^{n^{\prime}} \rho_{i}^{2}\left(\boldsymbol{q}^{\prime}\right)\right)^{-1} \rho_{x}\left(\boldsymbol{q}^{\prime}\right)\left(\tau-\rho_{x}\left(\boldsymbol{q}^{\prime}\right) F_{x}\right) .
$$

where $m$ is the slider mass, $G$ is the slider gravity component. The slider dynamics (7) can be also expressed as

$$
\ddot{x}=b(u+d)
$$


where $b=1 / m$ and

$$
\begin{aligned}
u & =\left(\sum_{i=1}^{n^{\prime}} \rho_{i}^{2}\left(\boldsymbol{q}^{\prime}\right)\right)^{-1} \rho_{x}\left(\boldsymbol{q}^{\prime}\right) \tau \\
d & =-\left[G+\left(\sum_{i=1}^{n^{\prime}} \rho_{i}^{2}\left(\boldsymbol{q}^{\prime}\right)\right)^{-1} \rho_{x}^{2}\left(\boldsymbol{q}^{\prime}\right) F_{x}\right] .
\end{aligned}
$$

Let $\boldsymbol{z}(t)=[x(t), \dot{x}(t)]^{\top}$, then (8) can be equivalently expressed as

$$
\dot{\boldsymbol{z}}(t)=\underbrace{\left[\begin{array}{ll}
0 & 1 \\
0 & 0
\end{array}\right]}_{\boldsymbol{A}} \boldsymbol{z}(t)+\underbrace{\left[\begin{array}{l}
0 \\
b
\end{array}\right]}_{\boldsymbol{B}}(u+d) .
$$

The control input $u$ must be transformed into joint space torque via the virtual work principle

$$
\tau=\rho_{x}\left(\boldsymbol{q}^{\prime}\right) u
$$

The main objective is to design a task-space controller such that the slider dynamics (10) follows the desired time-varying trapezoidal trajectory $\boldsymbol{z}_{\boldsymbol{d}}(t)=$ $\left[x_{d}(t), \dot{x}_{d}(t)\right]^{\top} \in \mathbb{R}^{2}$. The position error is $\boldsymbol{e}(t)=\boldsymbol{z}_{\boldsymbol{d}}(t)-\boldsymbol{z}(t)$. The closed-loop error dynamics is

$$
\begin{aligned}
\dot{\boldsymbol{e}}(t) & =\dot{\boldsymbol{z}}_{d}(t)-\boldsymbol{A} \boldsymbol{z}(t)-\boldsymbol{B}(u+d) \\
& =\boldsymbol{A} \boldsymbol{e}(t)-\boldsymbol{B}\left(u+d+\boldsymbol{B}^{\dagger} \boldsymbol{F}(t)\right)
\end{aligned}
$$

where $\boldsymbol{F}(t)=\boldsymbol{A} \boldsymbol{z}_{\boldsymbol{d}}(t)-\dot{\boldsymbol{z}}_{\boldsymbol{d}}(t)$ and $\boldsymbol{B}^{\dagger}$ denotes the pseudoinverse of $\boldsymbol{B}$.

\subsection{Optimal sliding surface design}

The main goal of this section is to design an optimal switching gain which ensures convergence of the closed-loop trajectories (12) into the next switching hyperplane

$$
s(\boldsymbol{e}(t))=\boldsymbol{C e}(t)
$$

where $C$ is the switching matrix gain. For sake of simplicity, the following coordinate transformation is considered

$$
\boldsymbol{\Psi}(t)=\boldsymbol{T} \boldsymbol{e}(t),
$$


where

$$
\boldsymbol{T}=\left[\begin{array}{c}
\left(\boldsymbol{B}^{\top}\right)^{\perp^{\dagger}} \\
\boldsymbol{B}^{\dagger}
\end{array}\right]
$$

where $\boldsymbol{B}^{\perp}$ represents the null space of matrix $\boldsymbol{B}$. The matrix transformation $\boldsymbol{T}$ is non singular due to the construction of matrix $\boldsymbol{B}$. The closed-loop error dynamics (12) under the new coordinates is

$$
\dot{\boldsymbol{\Psi}}(t)=\underbrace{\boldsymbol{T} \boldsymbol{A} \boldsymbol{T}^{-1}}_{\overline{\boldsymbol{A}}} \boldsymbol{\Psi}(t)+\underbrace{\boldsymbol{T} \boldsymbol{B}}_{\overline{\boldsymbol{B}}}\left(u+d+\boldsymbol{B}^{\dagger} \boldsymbol{F}(t)\right),
$$

where $\overline{\boldsymbol{A}}=\left[\begin{array}{ll}A_{11} & A_{12} \\ A_{21} & A_{22}\end{array}\right]$ and $\overline{\boldsymbol{B}}=\left[\begin{array}{l}0 \\ 1\end{array}\right]$. Hence the new system is

$$
\begin{aligned}
& \dot{\Psi}_{1}(t)=A_{11} \Psi_{1}(t)+A_{12} \Psi_{2}(t) \\
& \dot{\Psi}_{2}(t)=A_{21} \Psi_{1}(t)+A_{22} \Psi_{2}(t)+u+d+f(t),
\end{aligned}
$$

where $\boldsymbol{B}^{\dagger} \boldsymbol{F}(t)=[0, f(t)]^{\top}$. The switching hyperplane (13) is rewritten as

$$
s(\boldsymbol{\Psi}(t))=\boldsymbol{C} \boldsymbol{T}^{-1} \boldsymbol{\Psi}(t)=C_{1} \Psi_{1}(t)+C_{2} \Psi_{2}(t)=\overline{\boldsymbol{C}} \boldsymbol{\Psi}(t) .
$$

The term $C_{2}$ in (18) is set to $C_{2}=1$, then

$$
s(\boldsymbol{\Psi}(t))=C_{1} \Psi_{1}(t)+\Psi_{2}(t) .
$$

The existence of a sliding mode implies that $s(\boldsymbol{\Psi}(t))=\dot{s}(\mathbf{\Psi}(t))=0$ in finite time and the trajectories remain within the hyperplane. The sliding mode hyperplane is designed to minimize the following cost index

$$
J(\boldsymbol{e}(t))=\int_{t}^{\infty} \boldsymbol{e}^{\top}(\sigma) \boldsymbol{Q} \boldsymbol{e}(\sigma) d \sigma
$$

The above cost index is independent of the control input $u$. The cost index (20) is expressed in the new coordinates as

$$
J(\Psi(t))=\int_{t}^{\infty}\left(\Psi_{1}^{\top} Q_{11} \Psi_{1}+2 \Psi_{1}^{\top} Q_{12} \Psi_{2}+\Psi_{2}^{\top} Q_{22} \Psi_{2}\right) d \sigma,
$$

where $\boldsymbol{T} \boldsymbol{Q} \boldsymbol{T}^{-1}=\left[\begin{array}{ll}Q_{11} & Q_{12} \\ Q_{21} & Q_{22}\end{array}\right]$. The backstepping method [31] is used, where the following new virtual input is proposed

$$
v(t)=\Psi_{2}(t)+Q_{22}^{-1} Q_{12}^{\top} \Psi_{1}(t) .
$$


Then the first equation of (17) and the index (21) are rewritten as

$$
\begin{aligned}
\dot{\Psi}_{1}(t) & =\left(A_{11}-A_{22} Q_{22}^{-1} Q_{12}^{\top}\right) \Psi_{1}+A_{12} v(t) \\
J(\Psi) & =\int_{t}^{\infty}\left[\Psi_{1}^{\top}\left(Q_{11}-Q_{12} Q_{22}^{-1} Q_{12}^{\top}\right) \Psi_{1}+v^{\top} Q_{22} v\right] d \sigma \\
& =\int_{t}^{\infty}\left(\Psi_{1}^{\top}(\sigma) \bar{Q} \Psi_{1}(\sigma)+v^{\top}(\sigma) Q_{22} v(\sigma)\right) d \sigma,
\end{aligned}
$$

where $\bar{Q}=Q_{11}-Q_{12} Q_{22}^{-1} Q_{12}^{\top}$. Then, the virtual control solution [31] is obtained as in a linear-quadratic regulator (LQR) problem [32, 33]. The solution is

$$
v(t)=-Q_{22}^{-1} A_{12}^{\top} P \Psi_{1}(t)
$$

where $P$ is the solution of an Algebraic Ricatti equation (ARE) [32]. Finally, the optimal sliding hyperplane is

$$
\Psi_{2}(t)=-C_{1} \Psi_{1}(t)=-Q_{22}^{-1}\left(A_{12}^{\top} P+Q_{12}^{\top}\right) \Psi_{1}(t)
$$

\subsection{Second-order $S M C$}

The second-order SMC [24] used in this paper has the following structure

$$
\begin{aligned}
u & =k_{1} s_{x}, \quad k_{1}>0 \\
\dot{k}_{x} & =\operatorname{sign}(\sigma) \\
\sigma & =s(\boldsymbol{e}(t))-s\left(\boldsymbol{e}\left(t_{0}\right)\right) \exp ^{-\lambda\left(t-t_{0}\right)}, \quad \lambda>0 . \\
s_{x} & =\sigma+k_{2} k_{x}, \quad k_{2}>0
\end{aligned}
$$

The integral term in (30) overcomes the chattering problem. The exponential term in (29) is used to start on the sliding manifold and not from any other initial condition [34]. The closed-loop error dynamics (12) under the control (27) is

$$
\dot{\boldsymbol{e}}(t)=\boldsymbol{A} \boldsymbol{e}(t)-\boldsymbol{B}\left(k_{1}\left(\sigma+k_{2} \int_{t_{0}}^{t} \operatorname{sign}(\sigma) d \varsigma\right)+d+\boldsymbol{B}^{\dagger} \boldsymbol{F}(t)\right) .
$$

The next theorem states the stability and ultimate uniformly bounded (UUB) of the trajectories of the error dynamics (12) under the second-order SMC (27). 
Theorem 1. Consider the closed-loop error dynamics (31). The trajectories of (31) are UUB if the control gain $k_{1}$ satisfies

$$
k_{1}>\bar{\eta}+k_{0}
$$

where $\bar{\eta}$ is an upper bound of the unknown terms and $k_{0}>0$.

Proof 1. See Appendix A.

\subsection{Jacobian compensator}

The control law (27) must be transformed into control torque using (11). However, the Jacobian loses rank on the singularity points and therefore the controller loses controllability. In this paper, the following Jacobian compensator is used

$$
\widehat{\rho}_{x}=K_{\rho} \operatorname{sign}\left(\frac{\dot{x}}{\dot{q}}\right)
$$

where $K_{\rho}>0$ is the compensator gain. The sign of the compensator can be obtained on-line or off-line as in the switching criterion design. The gain can be chosen with the upper bound of the real Jacobian, that is, $K_{\rho}=\left|\max _{q} \rho_{x}(q)\right|$, or by manual tuning. The main advantage of the Jacobian compensator is that the controllability problem is avoided. For mote details refer to [10]. Furthermore, the Jacobian compensator does not affect the final output of the OSMC because the sliding gain $k_{1}$ can compensate the Jacobian modeling error.

\section{Experimental results}

Figure 5 shows the inverted Whitworth mechanism prototype used in this paper to test the proposed approach. The slider is located at the $Y$-axis so the $y$ variable is used instead of $x$. The mechanism is controlled by a permanent magnet motor of $12 \mathrm{~V}$. Angular position of the motor is measured by a BEI optical encoder. Resolution of the optical encoder is 2500 pulses per revolution and is directly coupled to the motor shaft. Cartesian position of the slider is measured by a US-digital strip sensor with a resolution of $300 \mathrm{cpi}$. 


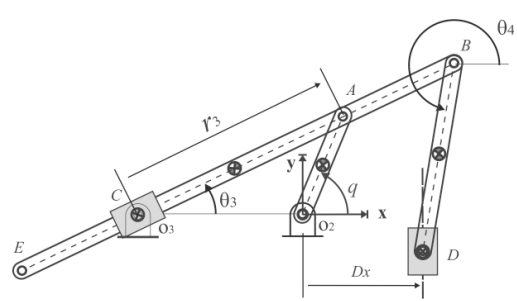

(a) Mechanism scheme

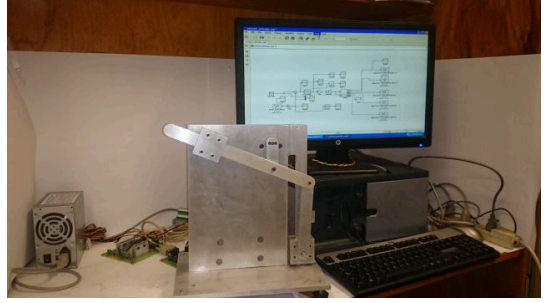

(b) Prototype

Figure 5: Inverted Whitworth mechanism

A Sensoray model 626 PCI data acquisition target endowed with inputs for optical encoders was used for the data acquisition. The data card electronics increases four times the optical encoder resolution up to 10,000 pulses per revolution.

The slider dynamics (7) was obtained from the extended dynamic model of Appendix B as

$$
m_{5} \ddot{y}+m_{5} g=u-\rho_{y} F_{y}
$$

where $m_{5}$ is the slider mass, $F_{y}=\left(\sum_{i=1}^{5} \rho_{i}^{2}\right)^{-1} \rho_{y} f_{y}, f_{y}$ is the contact force and $\rho_{y}$ is the slider Jacobian (see Appendix B). The dimensions of the mechanism prototype are: $O_{3 x}=-0.125 \mathrm{~m}, O_{3 y}=0 \mathrm{~m}, r_{2}=0.5 \mathrm{~m}, r_{4}=0.15 \mathrm{~m}$, $D_{x}=0.0705 \mathrm{~m}$ and $A B=0.08 \mathrm{~m}$.

\subsection{Tracking control}

First, the performance of the slider dynamics using the Jacobian compensator was tested. In this experiment there is no contact force, that is, $f_{y}=0$. The performance of the OSMC was compared against other linear and discontinuous controllers [35]. The main control objective was to force the mechanism slider position to follow the next desired reference

$$
y_{d}(t)=-0.2-0.2 \sin (\pi t) \text {. }
$$

The following controllers were used for comparison purposes: feedforwardfeedback controller (FFC), Linear Quadratic Tracking (LQT) control [13] and 
a second order SMC. All the controllers were designed in accordance to the slider-dynamics (34). The slider mass is $m_{5}=4.5 \times 10^{-4} \mathrm{~kg}$. In state-space, the slider dynamics (34) is written as

$$
\dot{\boldsymbol{z}}(t)=\left[\begin{array}{ll}
0 & 1 \\
0 & 0
\end{array}\right] \boldsymbol{z}(t)+\left[\begin{array}{c}
0 \\
2222.22
\end{array}\right](u(t)-0.00441)
$$

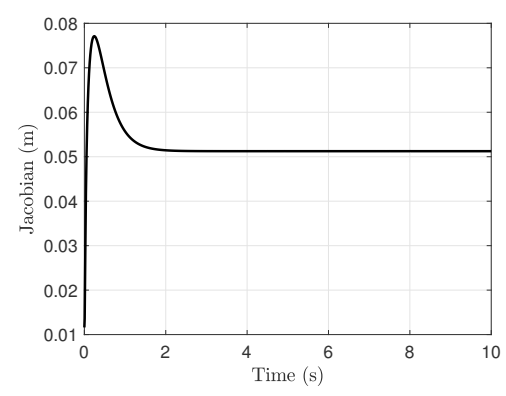

Figure 6: Jacobian $\rho_{y}\left(\boldsymbol{q}^{\prime}\right)$ at the return stroke

In contrast with the proposed OSMC, the FFC and the LQT controllers need a feedforward control law $u_{d}$ to guarantee position tracking and a feedback control law $u_{f}=-\boldsymbol{K} \boldsymbol{e}(t)$ to ensure stability, that is, $u=u_{d}+u_{f}$, for some gain matrix $\boldsymbol{K}^{1 \times 2}$. The feedforward control law is

$$
u_{d}=\boldsymbol{B}^{\dagger}\left(\dot{\boldsymbol{z}}_{d}(t)-\boldsymbol{A} \boldsymbol{z}_{\boldsymbol{d}}(t)\right) .
$$

For the FFC case, the stabilizing gain of the feedback control law was computed according to a desired performance as

$$
\operatorname{det}(\lambda \boldsymbol{I}-\boldsymbol{A}+\boldsymbol{B} \boldsymbol{K})=\lambda^{2}+2 \xi \omega_{n} \lambda+\omega_{n}^{2},
$$

where $\omega_{n}$ and $\xi$ denote the undamped natural frequency and the damping factor, respectively. The following desired performance was proposed: $\omega_{n}^{2}=1000$ and $\xi=1$.

The feedback control law of the LQT controller was obtained by minimizing the following cost function

$$
J(\boldsymbol{e}(t))=\int_{t}^{\infty}\left(\boldsymbol{e}^{\top}(\sigma) \boldsymbol{Q} \boldsymbol{e}(\sigma)+R u_{f}^{2}(\sigma)\right) d \sigma
$$


where $\boldsymbol{Q}=\boldsymbol{Q}^{\top}>0$ is the weight matrix of the tracking error and $R>0$ is the weight of the feedback control law. The solution of the LQT problem is obtained by solving an ARE of the closed-loop error dynamics (12) under the feedforward control (37) [32]. The next weight were proposed: $\boldsymbol{Q}=\boldsymbol{I}$ and $R=0.1$.

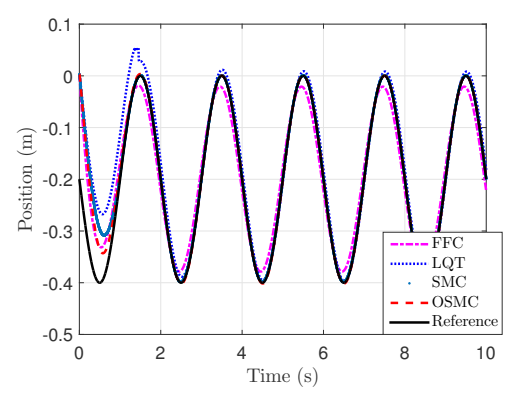

Figure 7: Position tracking results

The Ackermann-Utkin formula was used for the sliding hyperplane design of the second-order SMC, where $\lambda=-10$ was proposed as the desired pole. The main difference between this controller and the OSMC is the sliding hyperplane design. The following weight matrix $\boldsymbol{Q}=\operatorname{diag}\{1,0.1\}$ was used for the OSMC design. The final control gains of each controller were the following; FFC: $\boldsymbol{K}=[0.45,0.0285] ; \mathrm{LQT}: \boldsymbol{K}=[3.1623,3.1627] ;$ SMC: $\boldsymbol{C}=[0.0045,0.00045]$, $k_{1}=0.0004$ and $k_{2}=1$; OSMC: $\overline{\boldsymbol{C}}=[3.1623,1], k_{1}=0.00441$ and $k_{2}=1$. Figure 6 shows the performance of the slider Jacobian in the return stroke. The gain of the Jacobian compensator was set to $K_{\rho}=0.1$. At the return stroke, the slider velocity was negative, so $z=-1$.

Figure 7 shows the tracking results of each control law using the slider dynamics and the Jacobian compensator. It is well known that for good tracking results the feedforward control term must be designed accurately, otherwise the tracking results will be stable but with large error. SMC and OSMC overcome this issue by avoiding the feedforward control term.

The main advantage of using the slider dynamics (34) is that it only requires knowledge the slider mass instead of the complete mechanism dynamics which facilitates the control design. Its main disadvantage lies in in the feedforward 


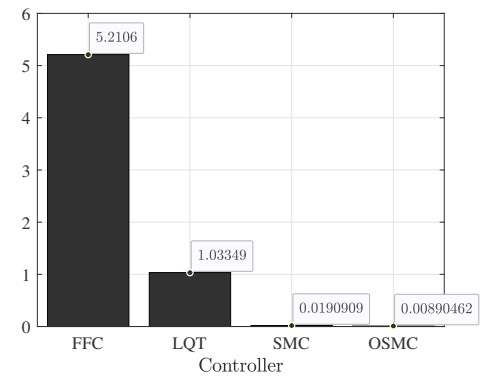

Figure 8: Mean squared error results.

controller of the FFC and LQT controllers, here stability of the closed-loop system can be guaranteed but not tracking convergence to the desired reference.

The mean squared error (MSE), $\bar{e}=\frac{1}{n} \sum_{i=1}^{n}\left(L e_{i}\right)^{2}$, of the last 2 seconds of the experiment was used in order to compare the controllers tracking precision. $L$ is a scaling factor whose value was proposed as $L=100$. The mean squared error results are given in Figure 8. Here it the reliability of the OSMC is shown, where the MSE was small in comparison to the other controllers and also guarantee optimal performance and robustness against disturbances.

\subsection{Constant cutting velocity task}

The gain of the optimal sliding hyperplane obtained in the last section was used in this experiment. The mechanism of Figure 5 was built without using a mechanism synthesis procedure. Therefore, the reciprocating method cannot be used for comparison purposes since it will not be a fair comparison.

The workpiece was of aluminum and it was proposed to use a greater contact force than the one proposed in the previous work [36] as

$$
f_{y}=\left\{\begin{array}{ll}
400 \mathrm{~N} & \text { if } \dot{y}<0 \text { and } y_{a} \leq y \leq y_{b} \\
0 \mathrm{~N} & \text { otherwise }
\end{array} .\right.
$$

The parameters and points of the velocity profile were obtained through the steps of Algorithm 1. The lengths of the workpiece and slider stroke were $L_{d}=0.3 \mathrm{~m}$ and $L_{c}=0.4667 \mathrm{~m}$, respectively. The constant cutting velocity 


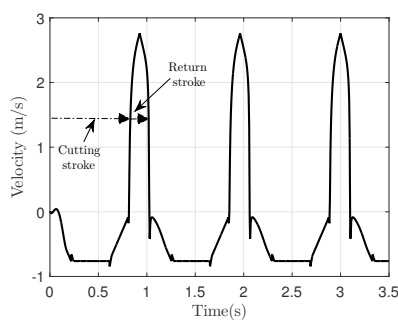

(a) Cutting velocity profile $\dot{y}$

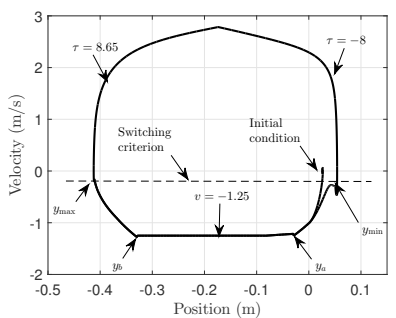

(b) Phase diagram: Position $y$ vs.

Velocity $\dot{y}$ of the slider block

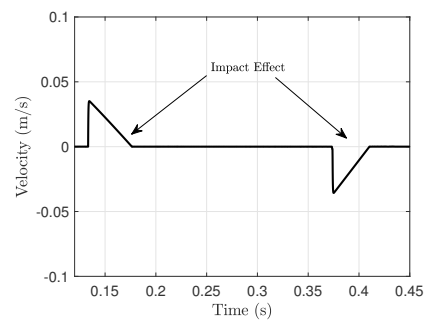

(c) Velocity error in the cutting stroke

Figure 9: Cutting velocity results

was proposed as $v=-1.25 \mathrm{~m} / \mathrm{s}$. The inverse kinematics solutions were: $q_{\min }=$ -0.729728 and $q_{\max }=-2.4119 \mathrm{rad}$. The mechanism forward kinematics (see Appendix B) is used to compute the singularity points as: $y_{\min }=0.05428$ and $y_{\max }=-0.4124 \mathrm{~m}$.

The times of the trapezoidal trajectory were: $t_{c}=0.3937 \mathrm{~s}$ and $t_{a}=t_{d}=$ $0.2187 \mathrm{~s}$. Finally the ends of the workpiece were located in: $y_{b}=-0.3291 \mathrm{~m}$ and $y_{a}=-0.0291 \mathrm{~m}$. The gains of the OSMC (27) were $k_{1}=40$ and $k_{2}=1$, which satisfy $k_{1} \geq\left\|\rho_{y} F_{y}+m_{5} g\right\|$. In Figure $9($ a) is shown the cutting velocity profile of three cutting cycles.

The return stroke was divided into two main parts to smooth the velocity response. The first part uses a positive torque of $\tau=8.65 \mathrm{Nm}$ and at the mean of the slider stroke is changed to the second part which uses a negative torque of $\tau=-8 \mathrm{Nm}$. Then the cutting task starts over again. 
Figure 9(b) shows the slider's phase diagram. The cutting stroke trajectory exhibits an accurate performance of the OSMC controller in the proposed intervals, that is, a constant velocity profile in the position interval $\left[y_{a}, y_{b}\right]$ and a smooth performance in the parabolic blends at the beginning position interval $\left[y_{\min }, y_{a}\right]$ and at the end position interval $\left[y_{b}, y_{\max }\right]$ of the cutting stroke. At the singularity point $y_{\max }$, the switching criterion changes the OSMC control law into fixed torque value to return the mechanism to $y_{\min }$ with high velocity amplitude. However, the switching criterion has a jerk effect which could produce damage to the mechanism bearings. This effect will be further analysed as future work. Since the Jacobian compensator was used, then the OSMC controller was not affected at the singularity points and shows a smooth transition between the cutting and return strokes.

Figure 9(c) shows the tracking velocity error $\dot{e}=\dot{y}_{d}-\dot{y}$ of the cutting stroke. Two small overshoots can be noticed which are caused by the interaction between the slider and the workpiece. The second order OSMC attenuates the contact force and ensures the constant velocity profile throughout the workpiece. These impact overshoots can be attenuated by increasing the sliding mode gains $k_{1}$ or $k_{2}$. Nevertheless, the jerk effect can be increased. Therefore, the exists a trade-off between the attenuation of the jerk effect and impact overshoots.

\section{Conclusions}

This paper addresses the uniform cutting problem of quick-return mechanisms. An OSMC controller was proposed to guarantee constant cutting velocity tracking and robustness against disturbances. The return stroke was controlled in open-loop by a fixed torque value in order to exploit the mechanical advantage, meanwhile the cutting stroke was controlled by the OSMC. A Jacobian compensator was used to avoid the singularities and controllability issues. The velocity profile was designed according to the length of the workpiece, constant velocity and the singularity points such that the reciprocating and mechanism synthesis methods were avoided. Experiments verify the approach with satis- 
factory results which also fills the current gap in real-applications of this kind of mechanisms using task-space controllers.

Future work will address the jerk problem caused by the switching criterion and how can be mitigated.

\section{Appendix A. Proof of Theorem 1}

Proof 2. The next Lyapunov function is considered

$$
V\left(s_{x}\right)=\frac{1}{2} s_{x}^{2}
$$

Its time derivative is

$$
\dot{V}\left(s_{x}\right)=s_{x}\left(\boldsymbol{C} \dot{\boldsymbol{e}}(t)+\lambda s\left(\boldsymbol{e}\left(t_{0}\right)\right) \exp ^{-\lambda\left(t-t_{0}\right)}+k_{2} \operatorname{sign}(\sigma)\right)
$$

Substituting (31) into $\dot{V}$ gives

$$
\dot{V}=s_{x}\left(\boldsymbol{C A} \boldsymbol{e}(t)-k_{1} s_{x}-d-\boldsymbol{B}^{\dagger} \boldsymbol{F}(t)-\lambda s\left(\boldsymbol{e}\left(t_{0}\right)\right) \exp ^{-\lambda\left(t-t_{0}\right)}-k_{2} \operatorname{sign}(\sigma)\right)
$$

Recall that $\boldsymbol{C B}=1$. The terms $|d| \leq \bar{d},\left|\lambda s\left(\boldsymbol{e}\left(t_{0}\right)\right) \exp ^{-\lambda\left(t-t_{0}\right)}\right| \leq \lambda\left|s\left(\boldsymbol{e}\left(t_{0}\right)\right)\right|$ and $\left\|\boldsymbol{B}^{\dagger} \boldsymbol{F}(t)\right\| \leq \bar{f}$ are bounded too for some positive scalars $\bar{d}$ and $\bar{f}$. Defining

$$
\|\boldsymbol{C A}\|\|\boldsymbol{e}(t)\|+\lambda\left|s\left(\boldsymbol{e}\left(t_{0}\right)\right)\right|+k_{2}+\bar{d} \leq \eta(t)
$$

where $\eta(t) \leq \bar{\eta}>0$ is a bounded function. So, $\dot{V}$ is reduced to

$$
\begin{aligned}
\dot{V} & \leq-k_{1} s_{x}^{2}+s_{x} \eta(t) \\
& \leq-\left|s_{x}\right|\left(k_{1}\left|s_{x}\right|-\bar{\eta}\right) .
\end{aligned}
$$

$\dot{V}$ is negative definite if $k_{1}$ satisfies (32), that is,

$$
\left|s_{x}\right|>\frac{\bar{\eta}}{k_{1}} \equiv \epsilon .
$$

Therefore, $s_{x}$ converges to a compact set $S_{\epsilon}$ of radius $\epsilon$ centered in $s_{x}=0$ as $t \rightarrow \infty$ and the tracking error trajectories remain bounded. 


\section{Appendix B. Inverted Whitworth mechanism model}

The inverted Whitworth mechanism scheme is shown in Figure 5(a). The independent coordinate is $q$ and the secondary coordinates are $\mathbf{s}=\left[r_{3}, \theta_{3}, \theta_{4}, y\right]^{\top}$. Its loop system equations are

$$
\begin{aligned}
& f_{1}(q, \mathbf{s})=r_{3} \cos \left(\theta_{3}\right)-r_{2} \cos (q)+O_{3 x}=0 \\
& f_{2}(q, \mathbf{s})=r_{3} \sin \left(\theta_{3}\right)-r_{2} \sin (q)+O_{3 y}=0 \\
& f_{3}(q, \mathbf{s})=O_{3 x}+\left(r_{3}+A B\right) \cos \left(\theta_{3}\right)+r_{4} \cos \left(\theta_{4}\right)-D_{x}=0 \\
& f_{4}(q, \mathbf{s})=O_{3 y}+\left(r_{3}+A B\right) \sin \left(\theta_{3}\right)+r_{4} \sin \left(\theta_{4}\right)-y=0
\end{aligned}
$$

The forward kinematics of (B.1) under the secondary coordinates $\mathbf{s}$ are

$$
\begin{aligned}
\theta_{3} & =\arctan \left(\frac{r_{2} \sin (q)}{r_{2} \cos (q)-O_{3 x}}\right) \\
r_{3} & =\frac{r_{2} \sin (q)}{\sin \left(\theta_{3}\right)} \\
\theta_{4} & =2 \pi-\arccos \left(\frac{D_{x}-O_{3 x}-\left(r_{3}+A B\right) \cos \left(\theta_{3}\right)}{r_{4}}\right) \\
y & =O_{3 y}+\left(r_{3}+A B\right) \sin \left(\theta_{3}\right)+r_{4} \sin \left(\theta_{4}\right)
\end{aligned}
$$

Consider the extended coordinates $\boldsymbol{q}^{\prime}=\left[q, r_{3}, \theta_{3}, \theta_{4}, y\right]^{\top}$. The extended Jacobian is obtained from the relation $\dot{\boldsymbol{q}}^{\prime}=\boldsymbol{\rho}\left(\boldsymbol{q}^{\prime}\right) \dot{q}$ as

$$
\left[\begin{array}{c}
\dot{q} \\
\dot{r}_{3} \\
\dot{\theta}_{3} \\
\dot{\theta}_{4} \\
\dot{y}
\end{array}\right]=\left[\begin{array}{c}
1 \\
-r_{2} \sin \left(q-\theta_{3}\right) \\
\frac{r_{2} \cos \left(q-\theta_{3}\right)}{r_{3}} \\
\frac{r_{2}\left(A B \sin \left(q-2 \theta_{3}\right)-\left(A B+2 r_{3}\right) \sin (q)\right)}{2 r_{3} r_{2} \sin \left(\theta_{4}\right)} \\
\frac{r_{2}\left(A B \sin \left(q-2 \theta_{3}+\theta_{4}\right)-\left(A B+2 r_{3}\right) \sin \left(q-\theta_{4}\right)\right)}{2 r_{3} \sin \left(\theta_{4}\right)}
\end{array}\right] \dot{q} .
$$

The mechanism extended dynamic is

$$
\begin{aligned}
& \boldsymbol{M}\left(\boldsymbol{q}^{\prime}\right)=\left[\begin{array}{lllll}
* & 0 & 0 & 0 & 0 \\
0 & * & 0 & * & 0 \\
0 & 0 & * & * & 0 \\
0 & * & * & * & 0 \\
0 & 0 & 0 & 0 & m_{5}
\end{array}\right]^{\top}, \boldsymbol{C}\left(\boldsymbol{q}^{\prime}, \dot{\boldsymbol{q}}^{\prime}\right)=\left[\begin{array}{lllll}
0 & 0 & 0 & 0 & 0 \\
0 & 0 & * & * & 0 \\
0 & * & * & * & 0 \\
0 & * & * & 0 & 0 \\
0 & 0 & 0 & 0 & 0
\end{array}\right] \\
& \boldsymbol{G}\left(\boldsymbol{q}^{\prime}\right)=\left[\begin{array}{lllll}
* & * & * & * & m_{5} g
\end{array}\right]^{\top}
\end{aligned}
$$

where $m_{5}$ is the slider mass and $*$ are not relevant terms for the control design. 


\section{References}

[1] Hsieh WH, Tsai CH. A study on a novel quick return mechanism. Transactions of the Canadian Society for Mechanical Engineering 2009;33(3):487500.

[2] Fung RF, Chen KW. Constant speed control of the quick return mechanism driven by a dc motor. JSME International Journal Series C Mechanical Systems, Machine Elements and Manufacturing 1997;40(3):454-61.

[3] Abdullah A, Chia L, Samad Z. The effect of feed rate and cutting speed to surface roughness. Asian Journal of Scientific Research 2010;3(4):278-87.

[4] Wu F, Zhang W, Li Q, Ouyang P. Integrated design and PD control of highspeed closed-loop mechanisms. J Dyn Sys, Meas, Control 2002;124(4):5228.

[5] Sun Y, Sun J, Li J, Xiong Q. An experimental investigation of the influence of cutting parameters on cutting temperature in milling ti6al4v by applying semi-artificial thermocouple. The International Journal of Advanced Manufacturing Technology 2014;70(5-8):765-73.

[6] Fata A. Temperature measurement during machining depending on cutting conditions. P\&A Science and Technology 2011;1(2):16-21.

[7] Loewen E. On the analysis of cutting-tool temperatures. Tras ASME 1954;76:217.

[8] Kus A, Isik Y, Cakir MC, Coskun S, Özdemir K. Thermocouple and infrared sensor-based measurement of temperature distribution in metal cutting. Sensors 2015;15(1):1274-91.

[9] Perrusquía A, Flores-Campos JA, Torres-Sanmiguel CR, González N. Task space position control of slider-crank mechanisms using simple tuning techniques without linearization methods. IEEE Access 2020;8:58435-42. 
[10] Flores Campos JA, Perrusquía A. Slider position control for slider-crank mechanisms with Jacobian compensator. Proceedings of the Institution of Mechanical Engineers, Part I: Journal of Systems and Control Engineering 2019;233(10):1413-21.

[11] Fung RF, Hwang CC, Huang CS, Chen WP. Inverse dynamics of a toggle mechanism. Computers \& structures 1997;63(1):91-9.

[12] Lin FJ, Wai RJ. Adaptive and fuzzy neural network slidingmode controllers for motor-quick-return servomechanism. Mechatronics 2003;13(5):477-506.

[13] Perrusquía A, Flores-Campos JA, Yu W. Simple optimal tracking control for a class of closed-chain mechanisms in task space. In: 2019 16th International Conference on Electrical Engineering, Computing Science and Automatic Control (CCE). IEEE; 2019, p. 1-6.

[14] Norton RL, et al. Design of machinery: an introduction to the synthesis and analysis of mechanisms and machines. Boston: McGraw-Hill Higher Education,; 2004.

[15] Yan HS, Chen WR. On the output motion characteristics of variable input speed servo-controlled slider-crank mechanisms. Mechanism and Machine Theory 2000;35(4):541-61.

[16] Vinu K, Ghosal A. Singularity analysis of closed-loop mechanisms and parallel manipulators. In: 15 th National Conference on Machines and Mechanisms. Citeseer; 2011, p. 1-9.

[17] Zheng E, Zhou X. Modeling and simulation of flexible slider-crank mechanism with clearance for a closed high speed press system. Mechanism and Machine theory 2014;74:10-30.

[18] Domínguez Ramírez OA, Parra Vega V, Díaz Montiel MG, Pozas Cárdenas MJ, Hernández Gómez RA. Cartesian sliding pd control of robot manipu- 
lator for tracking in finite time: Theory and experiments. DAAAM International, Viena Austria 2008;:257-72.

[19] Perrusquía A, Yu W. Task space human-robot interaction using angular velocity jacobian. In: 2019 international symposium on medical robotics (ISMR). IEEE; 2019, p. 1-7.

[20] García-Rodríguez R, Zegers P. Cartesian controllers for tracking of robot manipulators under parametric uncertainties. Robot Arms 2011;:109.

[21] Fateh MM, Farhangfard H. On the transforming of control space by manipulator jacobian. International Journal of Control, Automation, and Systems 2008;6(1):101-8.

[22] Perrusquía A, Yu W, Soria A. Position/force control of robot manipulators using reinforcement learning. Industrial Robot: the international journal of robotics research and application 2019;46(2):267-80.

[23] Fung RF, Chang CF. Force/motion sliding mode control of three typical mechanisms. Asian Journal of Control 2009;11(2):196-210.

[24] Perrusquía A, Yu W. Human-in-the-loop control using Euler angles. Journal of Intelligent \& Robotic Systems 2020;97(2):271-85.

[25] Yu W, Perrusquía A. Simplified stable admittance control using end-effector orientations. International Journal of Social Robotics 2020;12(5):1061-73.

[26] Yan HS, Chen WR. A variable input speed approach for improving the output motion characteristics of watt-type presses. International Journal of Machine Tools and Manufacture 2000;40(5):675-90.

[27] Fung R, Lin F, Wai R, Lu P. Fuzzy neural network control of a motorquick-return servomechanism. Mechatronics 2000;10(1-2):145-67.

[28] Gosselin C, Angeles J, et al. Singularity analysis of closed-loop kinematic chains. IEEE transactions on robotics and automation 1990;6(3):281-90. 
[29] Perrusquía A, Yu W, Soria A, Lozano R. Stable admittance control without inverse kinematics. IFAC-PapersOnLine 2017;50(1):15835-40.

[30] Ghorbel F. Modeling and PD control of closed-chain mechanical systems. In: Proceedings of 1995 34th IEEE Conference on Decision and Control; vol. 1. IEEE; 1995, p. 540-2.

[31] Flores-Campos JA, Perrusquía Guzmán J, Beltrán-Fernández JA, Hernández-Gómez LH. Sliding mode control of a water-displacement based mechanism applied to the orientation of a parabolic-trough solar concentrator. In: Defect and Diffusion Forum; vol. 370. 2016, p. 90-7.

[32] Perrusquía A, Yu W. Neural $\mathcal{H}_{2}$ control using continuous-time reinforcement learning. IEEE Transactions on Cybernetics 2020;.

[33] Mu C, Wang K, Sun C. Learning control supported by dynamic event communication applying to industrial systems. IEEE Transactions on Industrial Informatics 2020;17(4):2325-35.

[34] Mu C, He H. Dynamic behavior of terminal sliding mode control. IEEE Transactions on Industrial Electronics 2017;65(4):3480-90.

[35] Perrusquia A, Flores-Campos JA, Torres-San-Miguel CR. A novel tuning method of PD with gravity compensation controller for robot manipulators. IEEE Access 2020;8:114773-8.

[36] Gombi SL, Ramakrishna D. Estimation of impact effect on the cutting tool of a shaper from measurement of responses during machining. Journal for Manufacturing Science and Production 2012;12(3-4):161-9. 
2021-04-28

\title{
Optimal sliding mode control for cutting tasks of quick-return mechanisms
}

\author{
Perrusquía, Adolfo
}

\section{Elsevier}

Perrusquía A, Flores-Campos JA, Yu W. (2022) Optimal sliding mode control for cutting tasks of quick-return mechanisms. ISA Transactions, Volume 122, March 2022, pp. 88-95

https://doi.org/10.1016/j.isatra.2021.04.033

Downloaded from Cranfield Library Services E-Repository 